IAU Symposium

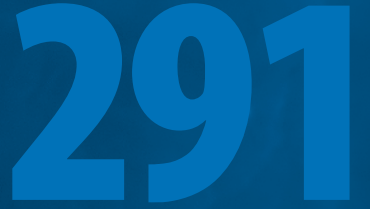

20 - 24 August 2012

Beijing, China

Proceedings of the International Astronomical Union

\title{
Neutron Stars and
}

Pulsars:

Challenges and

Opportunities

after 80 years

\section{Edited by}

\section{Joeri van Leeuwen}

ISSN 1743-9213

International Astronomical Union
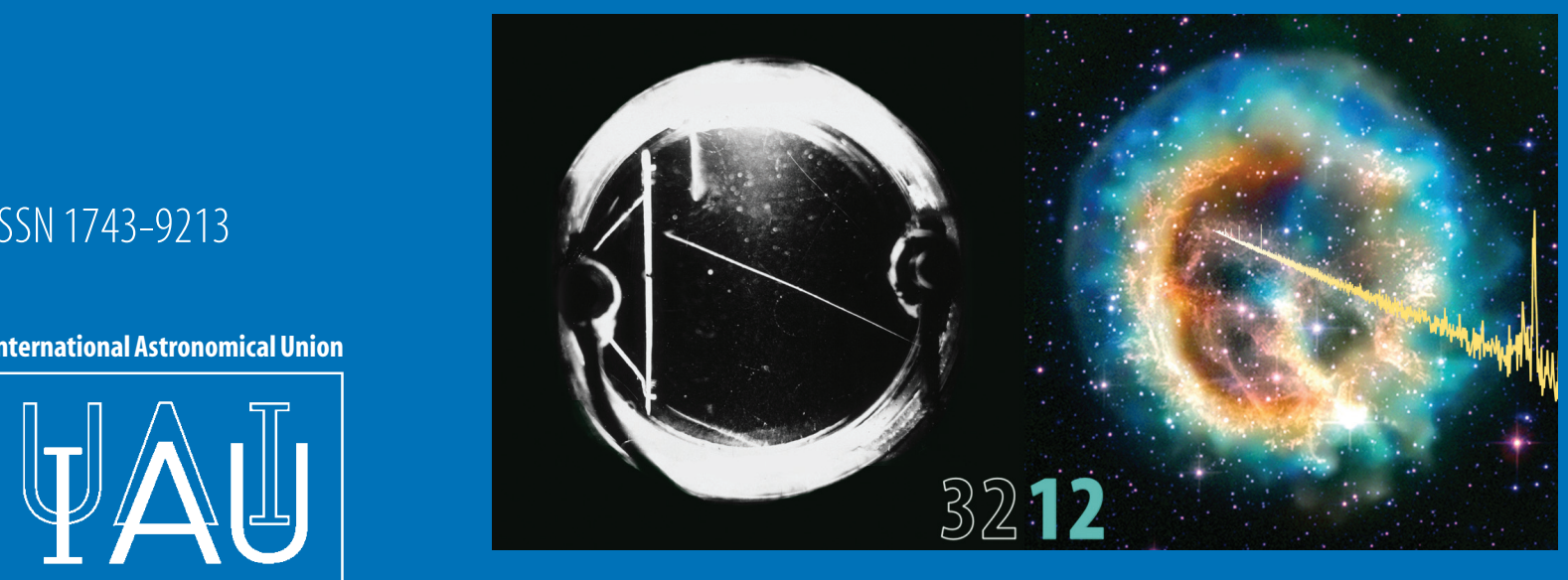

CAMBRIDGE UNIVERSITY PRESS 
NEUTRON STARS AND PULSARS:

CHALLENGES AND OPPORTUNITIES AFTER 80 YEARS

IAU SYMPOSIUM No. 291

COVER ILLUSTRATION:

$1932-2012:$

DISCOVERY OF THE NEUTRON - A NEUTRON STAR IN A SUPERNOVA

This diptych combines the 1932 detection of the neutron with the state of modern neutron-star and pulsar research, in 2012.

In the left-hand side photograph, neutrons have collided with the atoms in a layer of paraffin wax, ejecting a proton. The proton path is visible in the ionization chamber.

The right-hand panel shows an optical (HST) and X-ray (Chandra) false-color image of supernova remnant 1E 0102-7219. Overlaid for illustration is radio (WSRT) data of the Crab pulsar.

Left image courtesy of I. Joliot-Curie \& F. Joliot/NMSI. Right image of SNR E0102 courtesy of NASA/CXC/STScI/MIT/SOA/D.Dewey/J.DePasquale; overlay of Crab pulsar radio data courtesy of J. van Leeuwen/ASTRON. 


\title{
IAU SYMPOSIUM PROCEEDINGS SERIES
}

\author{
Chief Editor
}

THIERRY MONTMERLE, IAU General Secretary

Institut d'Astrophysique de Paris,

98bis, Bd Arago, 75014 Paris, France

montmerle@iap.fr

\section{Editor}

PIERO BENVENUTI, IAU Assistant General Secretary University of Padua, Dept of Physics and Astronomy, Vicolo dell'Osservatorio, 3, 35122 Padova, Italy piero.benvenuti@unipd.it 
INTERNATIONAL ASTRONOMICAL UNION

UNION ASTRONOMIQUE INTERNATIONALE

International Astronomical Union

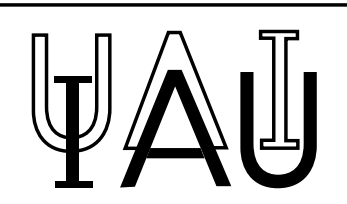

NEUTRON STARS AND

PULSARS:

CHALLENGES AND

OPPORTUNITIES

AFTER 80 YEARS

PROCEEDINGS OF THE 291st SYMPOSIUM OF THE INTERNATIONAL ASTRONOMICAL UNION HELD IN BEIJING, CHINA 20-24 AUGUST 2012

Edited by

JOERI VAN LEEUWEN

ASTRON, the Netherlands Institute for Radio Astronomy,

Postbus 2, 7990 AA, Dwingeloo, The Netherlands 
CAMBRIDGE UNIVERSITY PRESS

The Edinburgh Building, Cambridge CB2 2RU, United Kingdom

32 Avenue of the Americas, New York, NY 10013 2473, USA

10 Stamford Road, Oakleigh, Melbourne 3166, Australia

(C) International Astronomical Union 2013

This book is in copyright. Subject to statutory exception and to the provisions of relevant collective licensing agreements, no reproduction of any part may take place without the written permission of the International Astronomical Union.

First published 2013

Printed in the UK by MPG Books Ltd

Typeset in System $\mathrm{LT}_{\mathrm{EX}} 2_{\varepsilon}$

A catalogue record for this book is available from the British Library

Library of Congress Cataloguing in Publication data

This journal issue has been printed on FSC-certified paper and cover board. FSC is an independent, non-governmental, not-for-profit organization established to promote the responsible management of the worlds forests. Please see www.fsc.org for information.

ISBN 9781107033801 hardback

ISSN $1743-9213$ 


\section{Table of Contents}

Preface $\ldots \ldots \ldots \ldots \ldots \ldots \ldots \ldots \ldots \ldots \ldots \ldots \ldots \ldots \ldots \ldots \ldots \ldots \ldots \ldots \ldots \ldots \ldots \ldots$

Scientific Organizing Committee..................... xvii

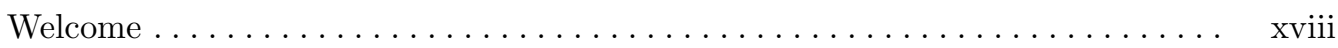

\section{Plenary Presentations}

Pulsars are cool. Seriously. . . . . . . . . . . . . . 3 S. M. Ransom

Magnetars: neutron stars with huge magnetic storms. . . . . . . . . . N. Rea

Probing gravitation with pulsars $\ldots \ldots \ldots \ldots \ldots \ldots \ldots \ldots \ldots \ldots \ldots \ldots$ M. Kramer

\section{Session 1: Pulsar Discovery I}

The High Time Resolution Universe surveys for pulsars and fast transients ... . M. J. Keith on behalf of the HTRU collaboration

The PALFA Survey: Going to great depths to find radio pulsars . . . . . . . . . P. Lazarus on behalf of the PALFA Collaboration

The hunt for new pulsars with the Green Bank Telescope............. R. S. Lynch, on behalf of the GBT $350 \mathrm{MHz}$ Drift-scan survey and Green Bank North Celestial Cap survey collaborations

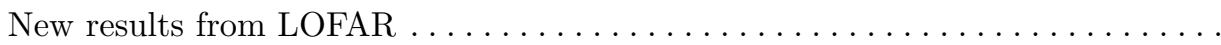

$V$. Kondratiev, on behalf of Ben Stappers and the LOFAR Pulsar Working Group

Conducting the deepest all-sky pulsar survey ever: the all-sky High Time Resolution

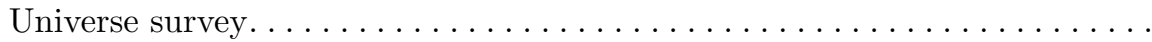
C. $\mathrm{Ng}$ and the HTRU Collaboration

A search for pulsars in the central parsecs of the Galactic center . . . . . . . . .

A. Siemion, M. Bailes, G. Bower, J. Chennamangalam, J. Cordes, P. Demorest, J. Deneva, G. Desvignes, J. Ford, D. Frail, G. Jones, M. Kramer, J. Lazio, D. Lorimer, M. McLaughlin, S. Ransom, A. Roshi, M. Wagner, D. Werthimer, \& R. Wharton

\section{Session 2: Pulsar Genesis - Neutron-star formation and birth properties}

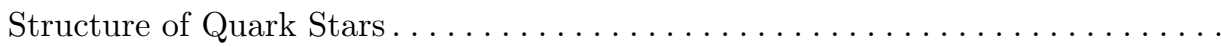

F. Weber, M. Orsaria, H. Rodrigues, \& S.-H. Yang

Numerical modeling of core-collapse supernovae and compact objects . . . . . . .

K. Sumiyoshi 
How "free" are free neutrons in neutron-star crusts and what does it imply for

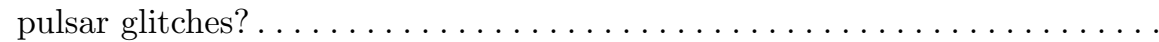

N. Chamel

Neutron star structure: what we learn from their masses and radii. . . . . . . . . F. $\ddot{O} z e l$

\section{Session 3: Pulsar Discovery II}

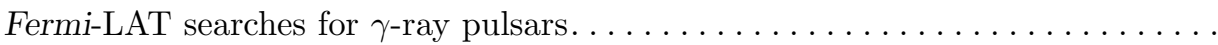

P. M. Saz Parkinson for the Fermi LAT Collaboration

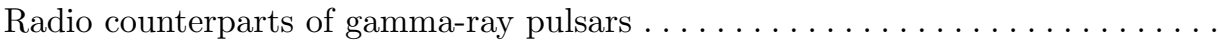

L. Guillemot, on behalf of the Fermi LAT Collaboration, the Fermi Pulsar Search Consortium and the Fermi Pulsar Timing Consortium

\section{Session 4: Pulsar Diversity}

Rotating Radio Transients and their place among pulsars ...........

S. Burke-Spolaor

Central compact objects and their magnetic fields $\ldots \ldots \ldots \ldots \ldots \ldots \ldots$

W. C. G. Ho

Discoveries of Rotating Radio Transients in the $350 \mathrm{MHz}$ Green Bank Telescope

Drift-scan Survey ............................... 107

C. Karako-Argaman and the GBT Drift-scan Collaboration

Pulsar searches in nearby dwarf spheroidal galaxies . . . . . . . . . . .

E. Rubio-Herrera \& T. Maccarone

The decaying magnetic field of magnetars: evidence and inference $\ldots \ldots \ldots \ldots$. . .

S. Dall'Osso

The first radio-quiet millisecond pulsar?

A. Belfiore

X-ray properties of rotation-powered pulsars. . . . . . . . . . . .

G. Pavlov $\&$ O. Kargaltsev

\section{Session 5: Binary Pulsars}

Binary pulsar evolution: unveiled links and new species............

A. Possenti

Surrounded by spiders! New black widows and redbacks in the Galactic field ...

M. S. E. Roberts

Formation of the planet orbiting the millisecond pulsar J1719-1438 . . . . . . .

L. M. van Haaften, G. Nelemans, R. Voss \& P. G. Jonker

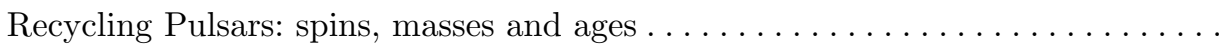

T. M. Tauris, M. Kramer \& N. Langer

A peculiar thermonuclear X-ray burst from the transiently accreting neutron star SAX J1810.8-260 . . . . . . . . . . . . . . . . . . . . . .

N. Degenaar 8 R. Wijnands 
Constraining neutron star EoS from cooling stages of X-ray bursts . . . . . . .

Neutron $\operatorname{star}$ masses. . . . . . . . . . . . . . . . . . . . .

D. Nice

\section{Session 6: Neutron star vibration and emission}

Merging neutron star binaries: equation of state and electrodynamics . . . . . . 149 D. Lai

Quasi-Periodic Oscillations in magnetars: linking variability and emission...... 155 C. D'Angelo

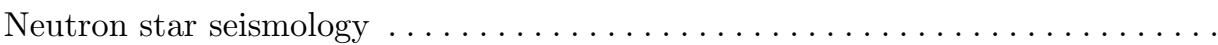

N. Andersson

Magnetar X-ray emission mechanisms

S. Zane

Long timescale radio emission variability and spin-down changes in PSR

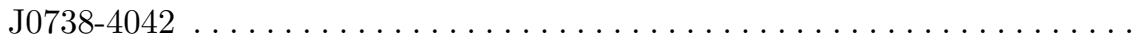

A. Karastergiou, P. Brook, S. Roberts, S. Buchner \&S S. Johnston

\section{Session 7: Pulsar timing and testing gravitational theories}

Pulsar Timing Arrays: Status \& Techniques . . . . . . . . . . . . . .

G. Hobbs

Prospects for probing strong gravity with a pulsar-black hole system. . . . . . .

N. Wex, K. Liu, R. P. Eatough, M. Kramer, J. M. Cordes E T. J. W. Lazio

Constraining the nanohertz gravitational wave background with the Parkes Pulsar

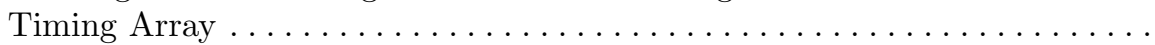

R. Shannon

Stochastic and continuous gravitational wave analysis pipelines for pulsar timing

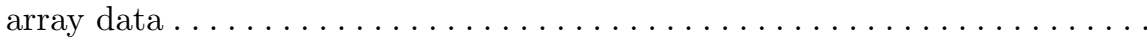

J. Ellis, F. Jenet, X. Siemens \& M. McLaughlin

19 Years of high precision timing of the millisecond pulsar J1713+0747 . . . . . W. Zhu

Update on the European Pulsar Timing Array .. 180 K. Liu

\section{Session 8: Pulsar Timing}

Timing noise and the long-term stability of pulsar profiles

A. Lyne 
Testing gravity theories in the radiative regime using pulsar timing arrays .....

K. J. Lee

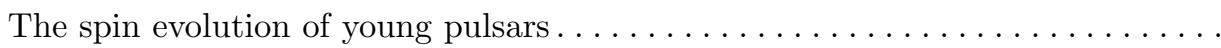

C. M. Espinoza

PSR J1906+0746: From relativistic spin-precession to beam modeling . . . . . . .

G. Desvignes, M. Kramer, I. Cognard, L. Kasian, J. van Leeuwen,

I. Stairs \& G. Theureau

The superslow pulsation X-ray pulsars in high mass X-ray binaries . . . . . . .

W. Wang

Vela Glitch Monitoring from HartRAO

S. Buchner

On the peculiarities in the spin-down of isolated radio pulsars $\ldots \ldots \ldots \ldots \ldots$ 208

A. Chukwude

\section{Session 9: Pulsars and the interstellar medium}

Galactic structure and turbulence, pulsar distances, and the intergalactic medium J. M. Cordes

Interstellar scattering - New diagnostics of pulsars and the ISM. . . . . . . J.-P. Macquart

Pulsars as excellent probes for the magnetic structure in our Milky Way....... J. Han

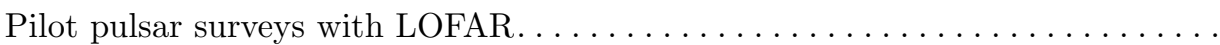

T. Coenen, on behalf of the LOFAR Pulsar Working Group

FRATs: Searching for fast radio transient in real-time with LOFAR . . . . . . . .

H. Falcke and the ILT LOFAR Consortium

\section{Session 10: Galactic distribution and evolution of neutron stars}

The Galactic Millisecond Pulsar Population

D. R. Lorimer

The pulsar population in Globular Clusters and in the Galaxy ........... P. C. C. Freire

Pulsar Wind Nebulae: On their growing diversity and association with highly mag-

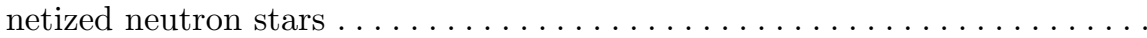

S. Safi-Harb

Constraining the luminosity function parameters and population size of radio

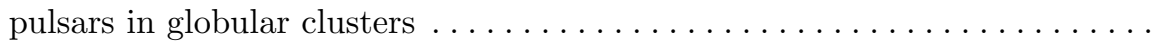

J. Chennamangalam, D. R. Lorimer, I. Mandel \& M. Bagchi

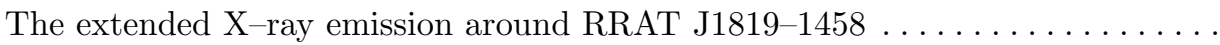

A. Camero-Arranz, N. Rea, M. A. McLaughlin, N. Bucciantini, P. Slane,

B. Gaensler, D. Torres, L. Stella, E. de Oña, G. Israel, F. Camilo $\mathscr{G}$

A. Possenti 
Diffusion and advection model for particle transport in young pulsar wind nebulae

$X$. Tang $\&$ R. A. Chevalier

Science with radio pulsar astrometry . . . . . . . . . . . . . .

S. Chatterjee

\section{Session 11: Pulsar magnetosphere and emission mechanisms}

The complex charm of the pulsar magnetosphere $\ldots \ldots \ldots \ldots \ldots \ldots \ldots$

A. N. Timokhin

The structure of the pulsar magnetosphere via particle simulation

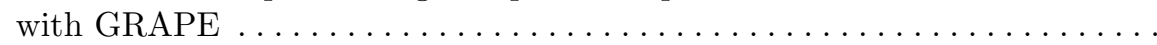

S. Shibata

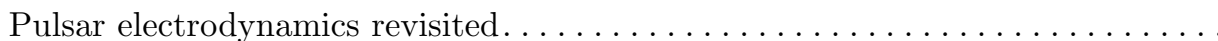

D. B. Melrose \& R. Yuen

Resistivity and dissipation in pulsar magnetospheres

J. Li, A. Spitkovsky, \& A. Tchekhovskoy

Modeling of pulsar magnetospheres $\ldots \ldots \ldots \ldots \ldots \ldots \ldots \ldots \ldots \ldots \ldots \ldots \ldots \ldots \ldots$

A. Spitkovsky

\section{Session 12: Emission mechanisms}

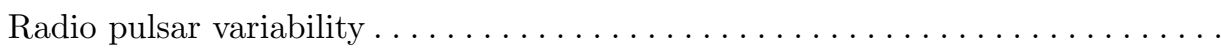

E. F. Keane

Elementary radiation patterns in pulsar profiles

J. Dyks \& B. Rudak

Pulsars in gamma rays: What Fermi is teaching us . . . . . . . . . . M. Kerr and the Fermi-LAT Collaboration

Binary pulsar B1259-63 spectrum evolution: detailed study. . . . . . . . . . M. Dembska, J. Kijak \& W. Lewadowski

Pulsar emission at the bottom end of the electromagnetic spectrum . . . . . .

V. Kondratiev and the LOFAR Pulsar Working Group

"An X-Raydio Switcheroo" - The detection of correlated mode changes

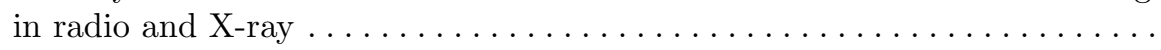

J. van Leeuwen, W. Hermsen, J. Hessels, L. Kuiper, D. Mitra, J. Rankin, B. Stappers \& G. Wright

A multi-wavelength campaign to study giant pulses from the Crab Pulsar ..... W. Majid

\section{Session 13: Future facilities}

The Five-hundred-meter Aperture Spherical radio Telescope project and its early

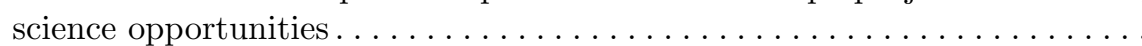

D. Li, R. Nan \& Z. Pan 
NuSTAR observations of rotation-powered pulsars and magnetars . . . . . . .

V. M. Kaspi, H. An, M. Bachetti, E. Bellm, A. M. Beloborodov, S. Boggs,

D. Chakrabarty, F. Christensen, B. Craig, F. Dufour, F. Harrison,

E. V. Gotthelf, T. Kitaguchi, C. Kouveliotou, K. Mori, M. Pivovaroff,

D. Stern, J. K. Vogel, W. Zhang, \& The NuSTAR Team

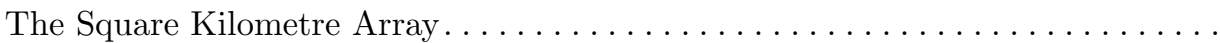

A. R. Taylor

\section{Summary}

Closing remarks

J. Bell Burnell

\section{Posters}

High magnetic field pulsars with magnetar-like activity $\ldots \ldots \ldots \ldots \ldots \ldots$

Y. Aoki

R-Process nucleosynthesis in high entropy environment in explosion of supernova

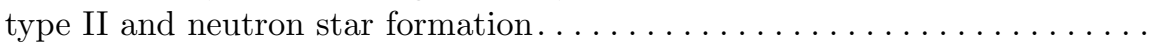

R. Baruah, K. Duorah, \& H. L. Duorah

A new low-B magnetar: Swift J1822.3-1606

A. Camero-Arranz, N. Rea, G. L. Israel, P. Esposito, J. A. Pons,

R. P. Mignani, R. Turolla, S. Zane, M. Burgay, A. Possenti, S. Campana,

T. Enoto, N. Gehrels, E. Göğüs, D. Götz, C. Kouveliotou, K. Makishima,

S. Mereghetti, \& S. R. Oates

Does a hadron-quark phase transition in dense matter preclude the existence of

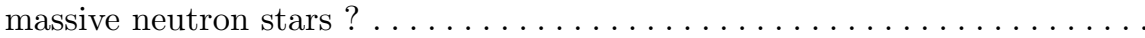

N. Chamel, A. F. Fantina, J. M. Pearson, \& S. Goriely

Unified description of dense matter in neutron stars and magnetars. . . . . . .

N. Chamel, R. L. Pavlov, L. M. Mihailov, Ch. J. Velchev, Zh. K. Stoyanov,

Y. D. Mutafchieva, M. D. Ivanovich, A. F. Fantina, J. M. Pearson, E

S. Goriely

Relativistic strange stars with anisotropy and B-parameter in pseudo spheroidal

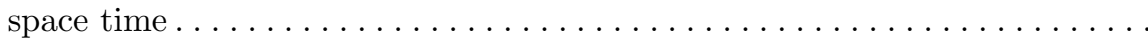

P. K. Chattopadhyay \& B. C. Paul

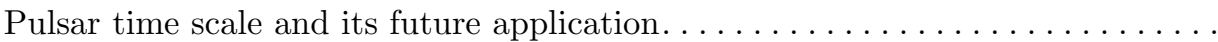

D. Chen, G. Hobbs, W. Coles, \& R. Manchester

New timing solutions for RRATs

B. Cui, J. Boyles, M. McLaughlin, \& N. Palliyaguru

Microlensing pulsars

S. Dai \& R. Xu

Constraints on Yukawa parameters by double pulsars $\ldots \ldots \ldots \ldots \ldots \ldots \ldots$

X.-M. Deng, Y. Xie, \& T.-Y. Huang 
SPAN512: A new mid-latitude pulsar survey with the Nançay Radio Telescope .

G. Desvignes, I. Cognard, D. Champion, P. Lazarus, P. Lespagnol,

D. A. Smith, \& G. Theureau

Annular gap model for multi-wavelength pulsed emission from young and

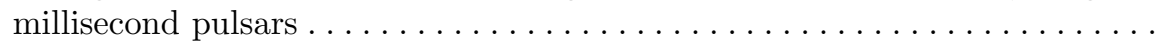

Y. J. Du\& G. J. Qiao

Characterizing glitches and timing irregularities in pulsars and magnetars .....

C. Espinoza, D. Antonopoulou, A. Patruno, B. Stappers, \& A. Watts

Can we see pulsars around Sgr $A^{\star}$ ? The latest searches with the Effelsberg

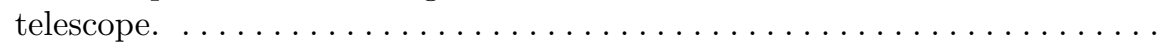

R. P. Eatough, M. Kramer, B. Klein, R. Karuppusamy, D. J. Champion,

P. C. C. Freire, N. Wex, \& K. Liu.

A survey of nulling pulsars using the Giant Meterwave Radio Telescope . . . . . .

V. Gajjar, B. C. Joshi, \& M. Kramer

The surface and inner temperatures of magnetars $\ldots \ldots \ldots \ldots \ldots \ldots$

Z. F. Gao, N. Wang, \& Q. H. Peng

Chandra observations of black widow pulsars ...

P. Gentile, M. McLaughlin, M. Roberts, F. Camilo, J. Hessels, M. Kerr,

S. Ransom, P. Ray, \& I. Stairs

Spin rotation, Chandler wobble and free core nutation of isolated multi-layer pulsars

A. Gusev \& I. Kitiashvili

Constraints of the compactness of the isolated neutron stars via X-ray

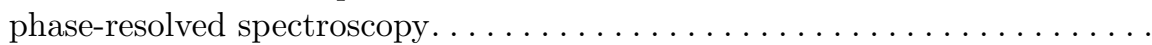

V. Hambaryan, V. Suleimanov, R. Neuhäuser, \& K. Werner

Magnetars are super hot and super cool

W. C. G. Ho, K. Glampedakis, \& N. Andersson

Birth accelerations of neutron stars $\ldots \ldots \ldots \ldots \ldots \ldots \ldots \ldots \ldots$

R. Heras

An XMM-Newton study of the supernova remnant G296.7-0.9 . . . . . . .

R. H. H. Huang, C. Y. Hui, L. Trepl, \& A. K. H. Kong

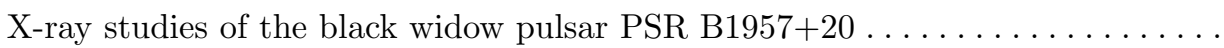

R. H. H. Huang, A. K. H. Kong, J. Takata, C. Y. Hui, L. C. C. Lin,

\& K. S. Cheng

Neutron stars: history of the magnetic field decay $\ldots \ldots \ldots \ldots \ldots \ldots \ldots$

A. P. Igoshev \& A. F. Kholtygin

Population synthesis of young neutron stars

A. P. Igoshev \& A. F. Kholtygin

Testing the relationship between nulling, drifting and mode-changing

B. C. Joshi 
Distribution of ionized gas density measured by differential VLBI Observations

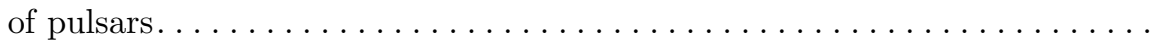

O. Kameya

Gamma-ray emission from pulsar binaries

J. Kirk \& I. Mochol

$\mathrm{TeV}$ cosmic-ray electrons from millisecond pulsars . .

S. Kisaka \& N. Kawanaka

Infrared AKARI observations of magnetars 4U 0142+61 and 1E $2259+586 \ldots \ldots$ T. Kohmura, K. Kaneko, S. Ikeda, M. Morii, K. Asano, M. Shirahara, \& N. Shibazaki

Magnetic field evolution in magnetars

Y. Kojima

Recoil velocity of pulsar/magnetar induced by magnetic dipole and quadrupole

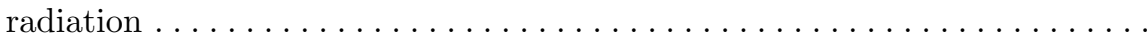

Y. Kojima

A pulsar census of the Local Group . . . . . . . . . . . . . . . .

V. Kondratiev, D. Lorimer, M. McLaughlin, \& S. Ransom

Tracking dispersion measure variations of timing array pulsars with the GMRT.

U. Kumar, Y. Gupta, W. van Straten, S. Ostowski, J. Roy, N. D. R. Bhat, M. Bailes, \& M. J. Keith

$H$-cluster stars

X. Y. Lai \& R. X. Xu

A data analysis library for gravitational wave detection. . . . . . . . . . .

A. Lassus, R. van Haasteren, C. M. F. Mingarelli, K. J. Lee, \& A. Vecchio

Modelling X-ray Pulse Profiles of Millisecond Pulsars . . . . . . . . . . .

D. A. Leahy, S. Morsink, \& W. Tian

Broadband spectral investigations of SGR J1550-5418 bursts. . . . . . . . . . .

L. Lin $\&$ E. Gögü̧

Profile stability and timing precision limit of millisecond pulsars $\ldots \ldots \ldots \ldots$. K. Liu

The missing compact star of SN1987A: a solid quark star? . . . . . . . .

X. W. Liu, J. D. Liang, R. X. Xu, J. L. Han, \& G. J. Qiao

Relativistic Cowling approximation for fluid oscillation modes of color supercon-

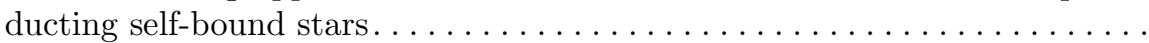

G. Lugones \& C. V. Flores

New observations of the Geminga pulsar at low radio frequencies........... V. M. Malofeev, O. I. Malov, S. V. Logvinenko, \& D. A. Teplykh

Do magnetars really exist? . . . . . . . . . . . . . . . . . . . . . .

I. F. Malov

Revisiting quark stars under the influence of strong magnetic fields . . . . . . . .

D. Menezes 
The slow X-ray pulsar SXP 1062 and associated supernova remnant in the Wing

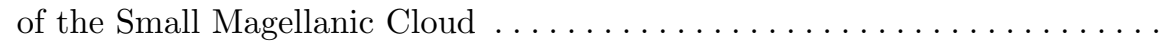

L. M. Oskinova, M. A. Guerrero, V. Hénault-Brunet, W. Sun, Y.-H. Chu,

C. Evans, J. S. Gallagher, III, R. A. Gruendl, \& J. Reyes-Iturbide

Seven pulsars in binary systems above the spin-up line $\ldots \ldots \ldots \ldots \ldots \ldots$

Y. Y. Pan \& N. Wang

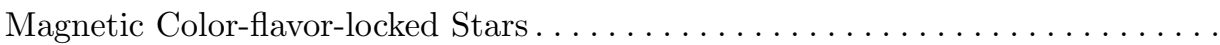

L. Paulucci, E. J. Ferrer, V. de la Incera, \& J. E. Horvath

Ultra-compact X-ray binaries with high luminosity: a key for a new scenario ... K. Pavlovskii \& N. Ivanova

Global structure of the pulsar force-free magnetosphere revisited . . . . . . .

S. Petrova

AXPs \& SGRs: Magnetar or Quarctar?...................

G. Qiao, X. Liu, R. Xu, Y. Du, J. Han, H. Tong, \& H. Wang

Searches for continuous gravitational waves with the LIGO and Virgo detector .

K. Riles, for the LIGO Scientific Collaboration and Virgo Collaboration

On the environments and progenitors of supernova remnants associated with highly

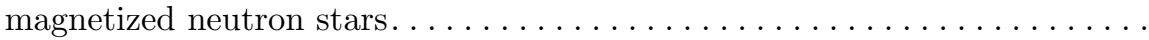

S. Safi-Harb \& H. S. Kumar

A high-energy catalogue of Galactic supernova remnants and pulsar wind nebulae S. Safi-Harb, G. Ferrand, \& H. Matheson

The new magnetar Swift J1822.3-1606 . . 486

P. Scholz, C.-Y. Ng, M. A. Livingstone, V. M. Kaspi, A. Cumming,

\& $R$. Archibald

X-ray properties of G308.3-1.4 and its central compact object . . . . . . . .

K. A. Seo, C. Y. Hui, R. H. H. Huang, L. Trepl, T.-N. Lu, A. K. H. Kong, \& F. M. Walter

Observations of transients and pulsars with LOFAR international stations and the

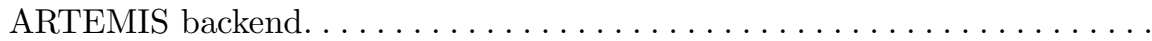
M. Serylak, A. Karastergiou, C. Williams, W. Armour, M. Giles, $\&$ the LOFAR Pulsar Working Group

Evidence for nonlinear and chaotic behaviour in pulsar spin-down rates . . . . . . A. Seymour \& D. Lorimer

New Constraints on Preferred Frame Effects from Binary Pulsars . . . . . . . . . . L. Shao, N. Wex, \& M. Kramer

Formation of Binary and Millisecond Pulsars: Puzzles and Possible Solutions... Y. Shao \& X.-D. Li

Detection of Giant pulses from pulsar PSR B0950+08 . . . . . . . . . . . T. V. Smirnova

Electric current diagnostics in the magnetosphere of neutron stars......... 505

A. V. Stepanov \& V. V. Zaitsev 


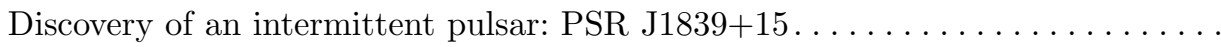

M. P. Surnis, B. C. Joshi, M. A. McLaughlin, \& V. Gajjar

Model of radio emission from spherically symmetric pulsar wind nebulae .....

S. J. Tanaka

Near IR Astrometry of Magnetars $\ldots \ldots \ldots \ldots \ldots \ldots \ldots \ldots \ldots \ldots \ldots$

S. P. Tendulkar

The spectral energy distributions of isolated neutron stars in the resonant cyclotron

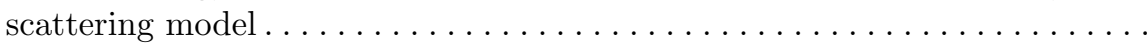

H. Tong \& R. Xu

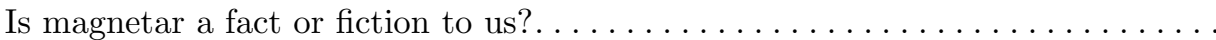

H. Tong \& R. X. Xu

What can Fermi tell us about magnetars? . . . . . . . . . . . . . . .

H. Tong \& R. X. Xu

Restrictions to neutron star models based on twin-peak quasi-periodic oscillations

G. Török, M. Urbanec, K. Goluchová, P. Bakala, E. Šrámková, \& Z. Stuchlik

Origin of the pulsar pulse fine structure.

O. M. Ulyanov, A. A. Seredkina, \& A. I. Shevtsova

Polarization sounding of the pulsar magnetosphere

O. M. Ulyanov, A. I. Shevtsova, \& A. A. Seredkina

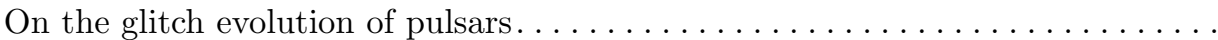

J. O. Urama, B. C. Joshi, \& A. E. Chukwude

Quadrupole moments of rotating compact stars

M. Urbanec, J. Miller, \& Z. Stuchlik

Particle simulation for an axisymmetric pulsar magnetosphere . . . . . . . .

T. Wada

Wave propagation in pulsar magnetospheres

C. Wang, D. Lai, \& J. Han

Search for the gravitational wave memory effect with the Parkes Pulsar Timing

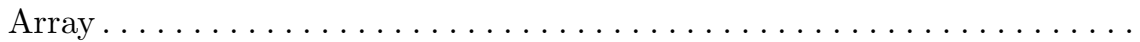

J. Wang, G. Hobbs, \& N. Wang

The distance indicators in gamma-ray pulsars. .

W. Wang

On the mode switching timescales of pulsar PSR B0329+54

H.-G. Wang, J.-L. Chen, Z.-G. Wen, \& F.-P. Pi

Curvature radiation in rotating pulsar magnetosphere

P. F. Wang, C. Wang, \& J. L. Han

Pulsed $\gamma$-ray emission from magnetar $1 \mathrm{E} 2259+586 \ldots \ldots \ldots \ldots \ldots \ldots$

J. H. K. Wu, C. Y. Hui, R. H. H. Huang, A. K. H. Kong, K. S. Cheng,

J. Takata, P. H. T. Tam, E. M. H. Wu, \& C.-Y. Liu 
Constraints on the standard model extension with binary pulsars $\ldots \ldots \ldots \ldots$

Y. Xie

Modeling pulsar time noise with long term decay modulated by short term oscillations of the magnetic fields of neutron stars $\ldots \ldots \ldots \ldots \ldots \ldots$ Y. Xie \& S. Zhang

VLBI astrometry of two millisecond pulsars $\ldots \ldots \ldots \ldots \ldots \ldots \ldots \ldots$

Z. Yan, Z.-Q. Shen, J.-P. Yuan, N. Wang, H. Rottmann, \& W. Alef

On the origin of the low-frequency QPO in GRS $1915+105 \rho$ state . . . . . . . S.-P. Yan, N. Wang, \& G.-Q. Ding

Rotation Measure variations for millisecond pulsars . . . . . . . . . . . 568 W. Yan, R. N. Manchester, \& N. Wang

Glitches detected in southern radio pulsars 571 M. $Y u$

Pulsar timing with the DFB at Nanshan

J. P. Yuan, N. Wang, Z. Y. Liu, \& J. B. Wang

FAST low frequency pulsar survey $\ldots \ldots \ldots \ldots \ldots \ldots \ldots \ldots \ldots \ldots \ldots \ldots \ldots \ldots \ldots$

Y. Yue, D. Li, \& R. Nan

Changes in Polarization Position Angle across the Eclipse in the Double Pulsar System. . . . . . . . . . . . . . . . . . . . . . . . . . . . . . 580

R. Yuen, R. N. Manchester, M. Burgay, F. Camilo, M. Kramer, D. B. Melrose, \& I. H. Stairs

Questions on accreting mass and minimum magnetic field of millisecond pulsars C. M. Zhang

Mutual influence of magnetic field decay and thermal evolution of rotational neutron stars . . . . . . . . . . . . . . . . . 586 X. Zhou, M. Kang, \& N. Wang 


\section{Preface}

The neutron hit the paraffin wax. It had been cast out when the alpha particle and beryllium nucleus had merged. In the wax, the neutron smashed out a proton. It traveled through the round bubble chamber, its mark captured on photographic plate. It's 1932, and the neutron is discovered.

A massive star exploded, and left a round supernova remnant. The neutron star was smashed out from the center of the crash. It spins and sends a beam of radio emission racing through the galaxy, to the telescope, arriving in 2012.

These two images, so visually similar as the front cover makes clear, mark the begin and current state of 80 years of neutron star research. That status, those newest results in neutron-star and pulsar studies, were presented at the IAUS291, Beijing, August 2012.

Several of the outstanding presentations in this volume are clearly linked to previous highlights in the lifetime of this octogenarian field. Chamel uses the sudden spin changes seen in some radio pulsars to constrain how the neutrons inside the star behave -80 years after the initial idea by Landau that such stars might exist. And while Baade and Zwicky proposed in 1934 that neutron stars form in supernovae, Sumiyoshi now presents our, partial, understanding of the mechanism driving these explosions. The new LOFAR discoveries presented by Kondratiev, and by Coenen, were made using a telescope operating at the same frequencies, and build as a similar array of dipoles, as the original Cambridge array with which Hewish and Bell found the first radio pulsar in 1967. Backer, much missed, found the first, isolated millisecond pulsar in 1982 - a discovery that echoed in Roberts' review on the now numerous detections of black-widow millisecond pulsars.

Other results, however, are exceedingly novel. Saz Parkinson presented tens of gammaray pulsars that were blindly detected with Fermi-LAT, uncovering a new population that is nearby and energetic, and often radio-quiet. An entire session, headed off by Hobbs, showcased the potential for gravity-wave pulsar astronomy. In several talks the intriguing new-found relations between spin-down and profile evolution were discussed. Burke-Spolaor, and Karako, explained how some radio pulsars only emit sporadically.

On August 24, 2012, 17:12 Beijing time, this IAU Symposium 291 came to a close. Yet, the talks and posters remain, in several complementary forms: as both slides and video onlinet, and as proceedings in the volume before you. 


\section{SCIENTIFIC ORGANISING COMMITTEE}

\section{Co-chairs:}

Dick Manchester

(Australia) Renxin Xu

(China)

\section{Members:}

Sarah Buchner

Jinlin Han

(South Africa) Yashwant Gupta

(India)

(China)

Rick Jenet

(USA)

Vicky Kaspi

(Canada)

Michael Kramer

Maura McLaughlin

(USA)

Andreas Reisenegger

(Germany)

Roger Romani

(USA)

Shinpei Shibata

(Chile)

Marten van Kerkwijk

(Canada) Joeri van Leeuwen

Nina Wang

(Japan)

(The Netherlands)

(UK) 
xviii

\section{Welcome}

The title of IAU Symposium 291 "Neutron Stars and Pulsars: Challenges and Opportunities after 80 Years" encapsulates the spirit of this Symposium: the 80 years since the idea of neutron stars was born and, in particular, the 45 years since pulsars were discovered have provided us with a rich harvest of scientific discovery, but many exciting avenues for future research remain. The Symposium, held in the huge Chinese National Convention Center adjacent to Beijing's Olympic Park as part of the 28th General Assembly of the International Astronomical Union, was very successful with more than 160 talks and posters presented over the five days and 14 sessions of the meeting. We are pleased that most of these presentations are represented in these Proceedings.

The sessions covered current searches for pulsars, both radio and gamma-ray, neutronstar formation and properties, binary pulsars, pulsar timing and tests of gravitational theories, magnetars, radio transients, radio, X-ray and gamma-ray pulse properties and emission mechanisms, and future facilities. This range of topics illustrates the diverse nature and wide application of pulsar research. Exciting new results were presented in all sessions and it is impossible to list them all. However, I would like to mention the three plenary talks, presented by Scott Ransom, Nanda Ray and Michael Kramer, which were outstanding and given to a standing-room-only audience despite the early hour. As the corresponding articles in this Proceedings show, they managed to successfully communicate the excitement of current pulsar research. I had many comments afterward, mostly from "non-pulsar" people, about how fascinating these talks were. I would also like to give special mention to Jocelyn Bell-Burnell's highly original closing remarks. We are grateful to her for allowing us to include them in this Proceedings.

Finally, I would like to give my thanks to the IAU and the GA Local Organising Committee for a well-run and successful meeting, to the Scientific Organising Committee for Symposium 291 for their assistance with putting together an excellent scientific programme, to all the presenters for realising the potential of the programme and to the Editor of the Proceedings, Joeri van Leeuwen, for all the hard work required to bring this volume to fruition. I hope and expect that it will be a valuable reference work for both current and future students and researchers in astronomy and astrophysics.

R. N. (Dick) Manchester

16 November, 2012 